\title{
Infirm Fitness
}

\section{Aaditya Kumar Sharma, Aman Ravi Lakra, Nishant Sharma, Punit Ekka}

UG Student, B. Tech, Computer Science and Engineering, Lovely Professional University, Phagwara, Punjab, India

\section{ABSTRACT}

\section{Article Info}

Volume 7, Issue 3

Page Number : 85-89

\section{Publication Issue :}

May-June-2021

\section{Article History}

Accepted : 02 May 2021

Published : 08 May 2021
Physically challenged people or the people with disabilities are those section of the society in which the society embarks no decision or gives any judgement about their physical abilities of the working body parts and the other requirements of them according to their physical fitness as well, the present generation has the awareness regarding keeping themselves fit and sportingly active but on the other hand the people who are physically challenged are unable to work on the same. The project hereby tries to implement the solution for the same by giving the various physical exercises that could be done by the physically challenges people according to their working body parts and according to their ability accordingly.

Keywords : Web Designing, Web Developers, Maintaining Fitness, Disabled, Physically Challenged, Categories of Disability, Fitness Reforms, Different Exercises for Different Body Parts.

\section{INTRODUCTION}

This project is entitled in order to make what actually the society and the people usually ignore and the situation regarding maintaining the physical fitness is left behind for the physically challenged people. This project work mostly aims to understand stability and instability dynamics of physically challenged people and how it affects fitness and how they can maintain their fitness as per their working body parts. There are different categories of physical as well as mental disabilities including various non-working body parts, semi working body parts, mental disability including postural deformities as well. So, basically the types of disabilities are generally affecting the persons movement, social relationships, vision, mental health, hearing, communication, thinking, learning and remembering abilities as well. The individuals having any kind of disability have been found $57 \%$ to be an obese most probably and hence its an important risk factor as any individual who is obese is most probably found to have chronic diseases or chronic conditions eg. Diabetes, stroke, heart disease or cancer and the only solution for the same are the aerobic physical exercises or activities such that the body can intake more amount of air and vice versa. Also the percentage of adults ages 18-64 who get no aerobic physical activity by disability type is $57 \%$ for Mobility, $40 \%$ for cognitive, $36 \%$ for vision, $33 \%$ for hearing and $26 \%$ for no disability which can be included in mental condition as well in which Mobility states serious difficulty in walking and climbing things like stairs, Cognitive is difficulty in remembering, concentrating and making decisions, Vision is having serious issues is seeing, even wearing spectacles and Hearing refers to the persons having 
hearing impairment. Physical Activity is described as any physical motion produced through skeletal muscles that require electricity expenditure. Activities undertaken at the same time as working, playing, travelling, sporting out house responsibilities and tasty in recreational interests are protected thereby. The WHO recommends for adults to do at least one hundred fifty mins of moderate-depth PA in the course of the week Compared to folks that meet the ones criteria, folks who are insufficiently bodily lively have a $20 \%$ to $30 \%$ elevated threat of allmotive mortality. When talking approximately or running with people with a incapacity it is critical to place the man or woman first. Disability does now no longer outline the man or woman as it's far handiest a scientific diagnosis. Positive language empowers and consequently inclusive language is used withinside the article and have to always be used.

"A incapacity is any circumstance of the frame or mind (impairment) that makes it extra tough for the man or woman with the circumstance to do sure activities (pastime limitation) and engage with the world round them (participation restrictions).

In the scientific version of incapacity the circumstance is regarded as a scientific and organic difficulty. Correcting ill-being is highlighted instead of stopping ill-being and selling well-being. On the contrary, in the social version incapacity is regarded as a distinction and now no longer judged. This version might emphasise obstacles consisting of structural elements or discriminatory behaviours that save you PA. Both the scientific and social version are criticised for highlighting severe factor of views. Out of it the social relational version become advanced which indicates that each impairments in addition to social and environmental obstacles can all perform simultaneously.

The project hereby focuses on developing an interface for the physically challenged people so that they can focus on the physical exercises or workout they need according to various categories of disabilities and various body parts available for movement for them. In the project, the interface is made accordingly such that the disabled person can themselves look after the various exercises that can be done in order to get maximum output and hence make the moving body parts fit. On the other hand the interface is available for the normal people as well and works like a information interface for them as well in which they can see various exercises for the various body parts and is like a training program which is designed in order to give adequate rest to the body parts which is been worked out and on the other hand focuses on the other body parts accordingly each day.

\section{RELATED WORK}

Firstly the group came in accordance to the group of people having various disability and hence decided to work on the same so that the people can get a little knowledge about the same and the people accessing the website will not only just access for themselves but also make they can ame the disable people around them aware of the same that it doesn't matter that they are not able to work with a particular body part but on the other hand, the set of exercises will also show them what needs to be done with correct posture and with number of repetitions and amount of weights accordingly.

The system can be improved by addition of monitoring features accordingly personalised for each person along with real-time monitoring and feedback sessions provided to the respective user, along with this the connection of fitness bands or any device which tracks the user calories can also be connected in order to get even more precise output and results of the personalised workouts designed accordingly for each person according to his/her requirements.

\section{DATA}

After the complete idea of the project was overall accepted by the group members, in this section it is 
described how the further processing of data was done.

A. The very first thing was to process that there is not just single type of disability amongst the people, there are various different types of disability or otherwise known as categories.

B. The second thing was to identify the disability by number of moving body parts, as due to any mishappening, because the person can have more than one disabled body parts, and that too different areas of body, eg. One is unable to work with hand other is unable to move legs and the other is unable to move both.

C. Coming next was to find out the physical movements to make the state of the body better and on the other hand to find the exercises for the different types of disability accordingly.

D. Proceeding to the previous one the next was to find out the technologies required by the group in order to make the required interface.

E. The required interface was required to be simple and with ease of access as well in order to give the best of the user-friendly experience so that whoever is using finds it easy to use the interface and to find out his/her required information from the interface.

F. The end was to coagulate all the data and studies found out till then and process it further so that all the things are compiled efficiently and is processed accordingly with the best of usage keeping in mind the requirement of every user visiting the interface.

\section{METHODOLOGY}

The basic methodology was to find out the types of disabilities. The following are the types of disabilities found:

A. Mobility - includes personnel with limited or no mobility in their respective body parts depending upon again the recoverable or not recoverable parts.

B. Cognitive - These are the persons having serious issues in the field of concentration, remembering or find hard to make decisions.

C. Vision - These are the personnel having issues in eyesight and is again categorised in serious or mild in which mild can be corrected using spectacles and on the other hand serious includes personnel who find it difficult to see even after spectacles or have no vision at all.

D. Hearing - these includes ones having difficulty in hearing or on the other hand we can say have hearing impairment.

E. Obesity - These are the overweight persons who have serious difficulty in movement, breathing and have lot more things included along with it.

\section{ANALYZING WEIGHT COACHING EXERCISES}

Analyzing weight training exercises are often enforced in two approaches. the primary approach is to check the skeletal movements of the trainee's performance with regard to the skeletal movements of a physical trainer of a similar exercise. The second approach is to observe angles of the joints of interest and the positions of the active joints. Among the 2 approaches, the first approach requires skeletal movements to be recorded for every exercise from a trainer then be compared to the trainee's performance. This comparison incorporates either statistic comparison algorithmic rule or a dynamic time warping algorithm. With such methods, however, time period feedback can't be provided. correct feedback should be provided in weight coaching exercises whenever the novice will a wrong movement. 


\section{ANALYZING MACHINE EXERCISES}

Analyzing machine exercises feature monitors machine exercises for the correctness while not a physical trainer's help. to trace machine exercises, accelerometer-equipped mobile devices are used. The mobile device ought to be placed on the moving a part of the machine. The mobile device and also the laptop are connected via $\mathrm{Wi}-\mathrm{Fi}$ to transfer acceleration knowledge in real-time. Acceleration data for a specific exercise is recorded from a physical trainer and hold on within the database. The patterns are recorded per the aim of the exercise. Then the user's acceleration or workout data for that exercise is compared and the factual or actual feedback/results and are given accordingly. The system counts the quantity of sets and repetitions in period to trace exercises. For a repetition, there are four stage points during a pattern of a graph. Initial position, peak position, minimum peak position and final position are the four main stages. once the pattern came up to the minimum peak stage, half repetition is completed and system will increase the number of repetitions by one.

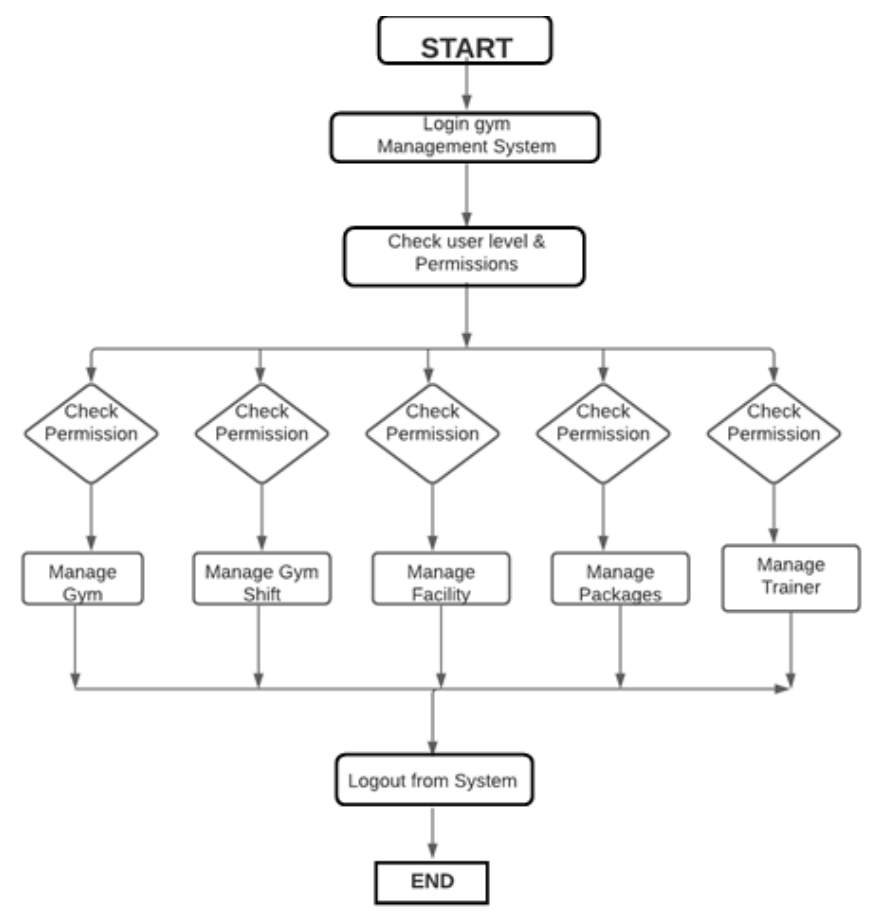

FIG 1. FLOW CHART OF WEBSITE
In this way as the workout are generally divided between bodybuilders, cross fitters and power lifters and in all these 3 categories only similarity is that all are working out and the difference between them is the type of workout these people in their respective categories are doing, the bodybuilders generally use external weights and machineries, on the other hand the cross fitters are generally limited to usage of body weights and powerlifters are in heavy weight lifting section.

\section{CONCLUSION}

In this paper, we have a tendency to delineated a system that has associate environment wherever folks will engage in physical exercises with the correct movements in their home. Exercises are monitored and analyzed mistreatment the angles and therefore the positions of the active angles. for every exercise, the right techniques were implemented using the relevant angles of the active joints and/or the positions of the active joints. we have a tendency to derived a few of algorithms to count exercise repetitions and to check two acceleration information sequences.

There are few limitations in the system. For example, Fitness Mate doesn't establish that exercise is performed by the user. The system monitors the exercises assumptive that the user performs the exercise that's displayed on the system. Currently, the system can't be deployed in an exceedingly gymnasium surroundings wherever multiple users are to use the system at the same time. Implementing the flexibility to handle multiple effort sessions from multiple users simultaneously is planned for future work.

The interface successfully delivers the basic exposure to the various exercises according the disability of a particular person and also works well for the person 
with no disability as well and gives workouts for every body part, with tutorial as well so that no correction required or doubt in the mind of the user regarding the posture or the way in which the workout is to be done.

\section{REFERENCES}

[1]. "Types of Physical Activity" Available: http://www.mindingourbodies.ca/toolkit/gettin g_started/types_of_physical_activity Accessed: Sep. 27, 2016

[2]. "Your Online Guide to Weight Training Exercises" Available: http://www.weighttraining-exercises.com/ Accessed: Sep. 26, 2016.

[3]. A. Mobini, S. Behzadipour, and M. S. Foumani, "Accuracy of Kinect's skeleton tracking for upper body rehabilitation applications," Disability and Rehabilitation: Assistive Technology, no. 0, pp. 1-9, 2013.

[4]. "Kinect for Windows Sensor Components and Specifications" Available: https://msdn.microsoft.com/enus/library/jj131033.aspx Accessed: Sep. 27, 2016.

[5]. N. Ravi, N. Dandekar, P. Mysore, and M. L. Littman, "Activity recognition from Accelerometer data," In Proc. 17th conference on Innovative applications of artificial intelligence, 2005, pp. 1541-1546.

[6]. "SmartSpot," Online. Available: https://www.smartspot.ioAccessed: Aug. 20, 2016.

[7]. D. Haas, S. Phommahavong, J. Yu, S. KrügerZiolek, K. Möller, and J. Kretschmer, "Kinect based physiotherapy system for home use," In Proc. Current Directions in Biomedical Engineering, 2015, pp. 180-183.

[8]. K. Chang, M. Y. Chen and J. Canny, "Tracking free-weight exercises," In Proc. 9th
International conference on ubiquitous computing, UbiComp '2007, pp. 19-37.

[9]. D. Monahan, D. Juall, "Fitness applications and devices," fitlinxx.net, para. 1993.Online. Available: http://www.fitlinxx.net/ Accessed Feb. 27, 2016

[10]. "Review: Nike+ Kinect is the perfect exercise game, with a fatal flaw" Available: https://www.fastcodesign.com/1671136/review -nike-kinect-is-the-perfect-exercise-gamewith-a-fatal-flawAccessed: Aug. 23, 2016.

[11]. D. Anton, A. Goni, A. Illarramendi, J. J. TorresUnda and J. Seco," KiReS: A Kinect-based telerehabilitation system," presented at 15th IEEE International Conference on e-Health Networking, Applications and Services (Healthcom 2013), 2013.

[12]. S. Ganesan, L. Anthony, "Using the Kinect to encourage older adults to exercise: a prototype," International Conference on Human Factors in Computing Systems '05, May, 2012, pp. 2297-2302.

[13]. Asselin, Ortiz, Pui, Smailagic, Kissling, "Implementation and evaluation of the personal wellness coach," In Proc. 5th International Workshop on Smart Application and Wearable Computing, 2005, pp. 529-53

\section{Cite this article as :}

Aaditya Kumar Sharma, Aman Ravi Lakra, Nishant Sharma, Punit Ekka, "Infirm Fitness", International Journal of Scientific Research in Computer Science, Engineering and Information Technology (IJSRCSEIT), ISSN : 2456-3307, Volume 7 Issue 3, pp. 85-89, May-June 2021. Available at doi : https://doi.org/10.32628/CSEIT217316 Journal URL : https://ijsrcseit.com/CSEIT217316 